Musées, Patrimoine et Culture scientifiques et techniques

$141 \mid 2012$

mai - juin 2012

\title{
Exposition et conservation d'une peau sub-fossile : le cas du mammouth de Lyakhov
}

Exhibition and conservation of a sub-fossil hide: the case of the Lyakhov mammoth

Jean-Guy Michard et Amandine Péquignot

\section{(2) OpenEdition}

Journals

Édition électronique

URL : http://journals.openedition.org/ocim/1068

DOI : 10.4000/ocim.1068

ISSN : 2108-646X

Éditeur

OCIM

Édition imprimée

Date de publication : 1 mai 2012

Pagination : 24-32

ISSN : 0994-1908

Référence électronique

Jean-Guy Michard et Amandine Péquignot, «Exposition et conservation d'une peau sub-fossile : le cas du mammouth de Lyakhov », La Lettre de I'OCIM [En ligne], 141 | 2012, mis en ligne le 01 mai 2014, consulté le 30 avril 2019. URL : http://journals.openedition.org/ocim/1068 ; DOI : 10.4000/ocim.1068 


\title{
Exposition et conservation d'une peau sub-fossile : le cas du mammouth de Lyakhov
}

\author{
Jean-Guy Michard et Amandine Péquignot *
}

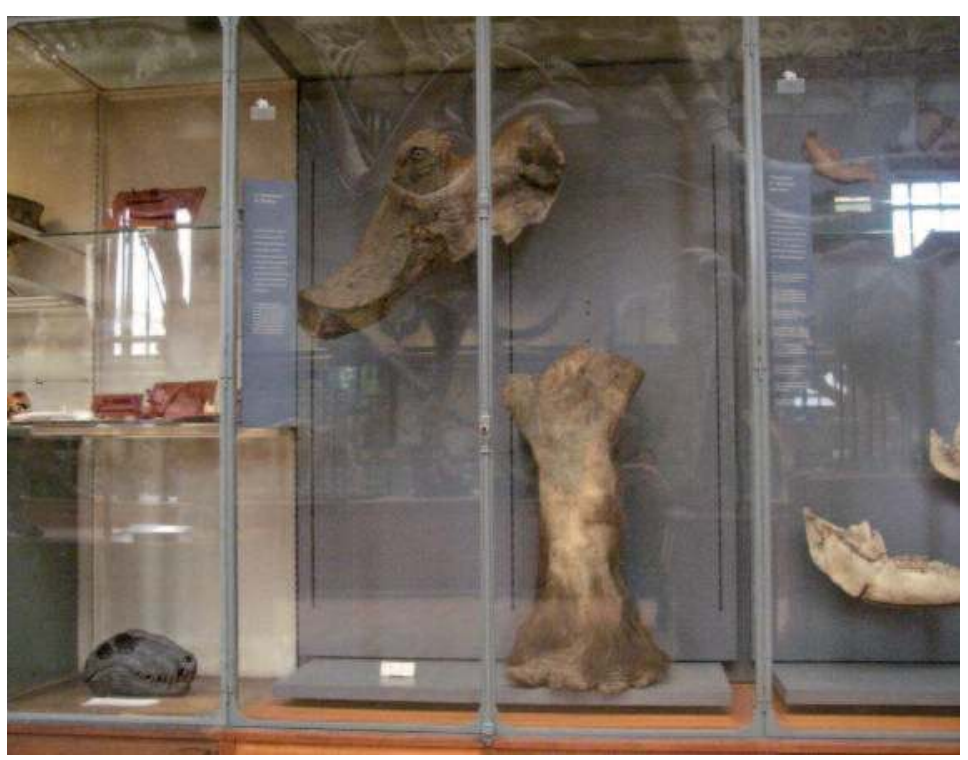

La tête et une patte postérieure gauche du mammouth laineux (Mammuthus primigenius) de Lyakhov sont conservées au Muséum national d'Histoire naturelle depuis 1912, et exposées dans la galerie de Paléontologie depuis 1998.

() A. Péquignot

* Jean-Guy Michard est maître de conférences au Muséum national d'Histoire naturelle, centre de recherche sur la conservation des collections michard@mnhn.fr Amandine Péquignot est maître de conférences au

Muséum national d'Histoire naturelle, centre de recherche sur la conservation des collections apeq@mnhn.fr
Après avoir rappelé le parcours du mammouth de Lyakhov depuis son arrivée au Muséum national d'Histoire naturelle il y a un siècle, les auteurs analysent les facteurs d'altération potentiels - liés aux conditions d'éclairage, de température et d'hygrométrie de ce spécimen et plus généralement précisent les précautions particulières qui doivent entourer l'exposition et la conservation des peaux de mammifères sub-fossiles.

Depuis 1998, la tête et une des pattes du mammouth laineux dit « de Lyakhov » ou " de Vollossovitch » sont exposées au public dans la galerie de Paléontologie du Muséum national d'Histoire naturelle, parmi les autres proboscidiens fossiles. Tous ces objets sont soumis sans distinction aux mêmes conditions de conservation. Or, de par sa nature séchée ou pseudo tannée, la patte de Lyakhov nécessite des paramètres adaptés et différents de ceux requis pour les fossiles. À l'époque de cette étude (2007-2008), aucune réflexion et adaptation n'ont été engagées pour ce cas particulier. Or, les facteurs environnementaux (climatique et physique, biologique et géographique) sont sources de dégradation des collections lorsqu'ils ne sont pas adaptés. La patte du mammouth laineux de Lyakhov présente un état de dégradation avancé et assez préoccupant. La première observation est la perte des phanères; les $4 / 5 \mathrm{e}$ de la surface de la peau sont totalement dégarnis. Cette zone présente, par ailleurs, des craquelures, pelures avancées et des altérations physiques irréversibles 
demanda à Yves Coppens d'en achever le montage, en y ajoutant un moulage des os des mains et des pieds. Une armature métallique moins visible que celle réalisée quinze ans auparavant avait été usinée et le spécimen fut présenté dans l'exposition temporaire L'Évolution des Vertébrés et l'Origine de l'Homme qui se tenait au rez-de-chaussée du bâtiment de Cryptogamie-Phanérogamie (Coppens, 1958). Ce squelette remonté rejoignit ensuite le hall de la galerie d'Anatomie comparée et de Paléontologie, dont il ne bougera qu'en de très rares occasions. En 1998, lors des réaménagements muséographiques effectués pour l'exposition de préfiguration de la rénovation de la galerie d'Anatomie comparée et de Paléontologie, Ossements, ce spécimen prendra sa place actuelle au premier étage du bâtiment, avec les autres proboscidiens fossiles (Van Praët, 1999) et une vitrine murale accueillera la partie gauche de la tête reconstituée avec la peau originale et l'extrémité de la patte postérieure gauche. Le squelette et cette patte traverseront le Jardin des plantes pour être présentés dans la Grande Galerie de l'Évolution lors de l'exposition temporaire Au temps des mammouths de mars 2004 à janvier 2005.

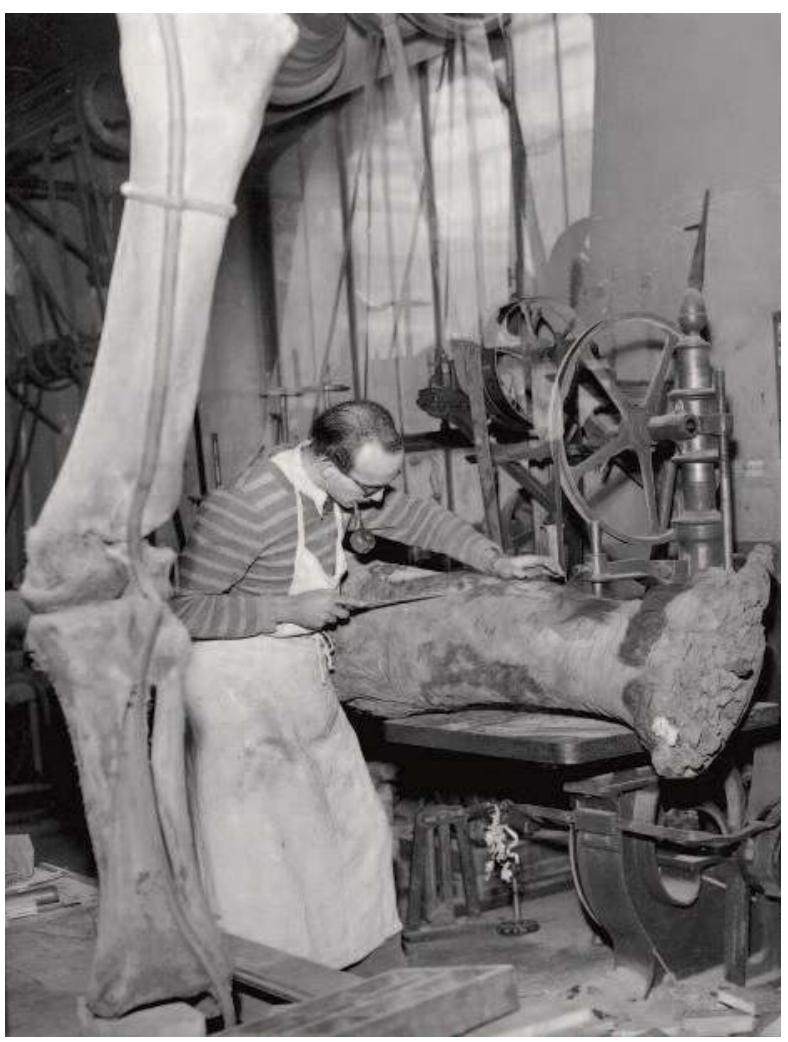

Jacques Richir, préparateur au laboratoire de Paléontologie (MNHN), en train de travailler sur l'une des pattes du mammouth de Vollossovitch, à côté du premier montage du squelette (cliché aimablement communiqué par Philippe Richir). (๐) International News Photos
En revanche, l'historique des traitements appliqués, des différents lieux de conservation ou des mises en exposition des parties molles du mammouth de Vollossovitch est nettement moins bien connu. Les plus anciennes photographies des parties molles datent vraisemblablement de l'arrivée du spécimen à Paris. La patte postérieure gauche montre une toison encore abondante sur presque toute sa hauteur, mais lors de son installation dans la galerie d'Anatomie comparée et de Paléontologie en 1998, ce spécimen avait déjà perdu une grande partie de son pelage, vraisemblablement à la suite d'expositions temporaires, et/ou de conditions de conservation et de manipulation malheureuses. Nous avions pu constater alors que les fragments de peau et certaines autres parties molles, étaient rangés dans un placard extrêmement chaud et sec d'une pièce en sous-sol du bâtiment de Paléontologie. Selon le chargé de conservation de la collection des mammifères fossiles (P. Tassy, communication personnelle), ces spécimens y étaient entreposés depuis longtemps. Nous ne savons que peu de choses sur les éventuelles présentations au public de ces restes. Une photographie prise entre 1945 et 1950, montre Jacques Richir, préparateur du laboratoire de Paléontologie, restaurant une autre de ces extrémités ; ce qui ne veut pas dire qu'elle fut exposée ensuite. Les documents décrivant la découverte de ce spécimen nous apprennent que la dépouille gisait sur le flanc gauche, et donc que cette partie de l'animal était encore protégée dans le permafrost. En 1994, l'extrémité antérieure gauche sera mise en vitrine dans la Grande Galerie de l'Évolution où elle demeure actuellement ; l'extrémité postérieure droite est en réserve, tandis que l'extrémité antérieure droite est parfois prêtée à l'extérieur du Muséum (Tassy, 2007).

Nous ne possédons aucune information sur l'éventualité d'un tannage, ou pour le moins d'un traitement conservateur, des fragments de peau, ou des éléments anatomiques associés à de la peau. Cependant, des analyses XFR non destructives (portable XRF Niton XLt 700), effectuées en de nombreux points de la tête et de la patte exposées ont révélé la présence d'arsenic et de fer. Des résultats identiques ont été observés lors de l'analyse de la patte antérieure gauche exposée dans la Grande Galerie de l'Évolution. De nombreux clous et agrafes sont présents dans ces trois spécimens, mais les spots réalisés en dehors des zones contenant ces objets métalliques, indiquent malgré tout la présence de fer dans des proportions à peu près identiques. Seule une analyse chimique destructive aurait permis d'interpréter 


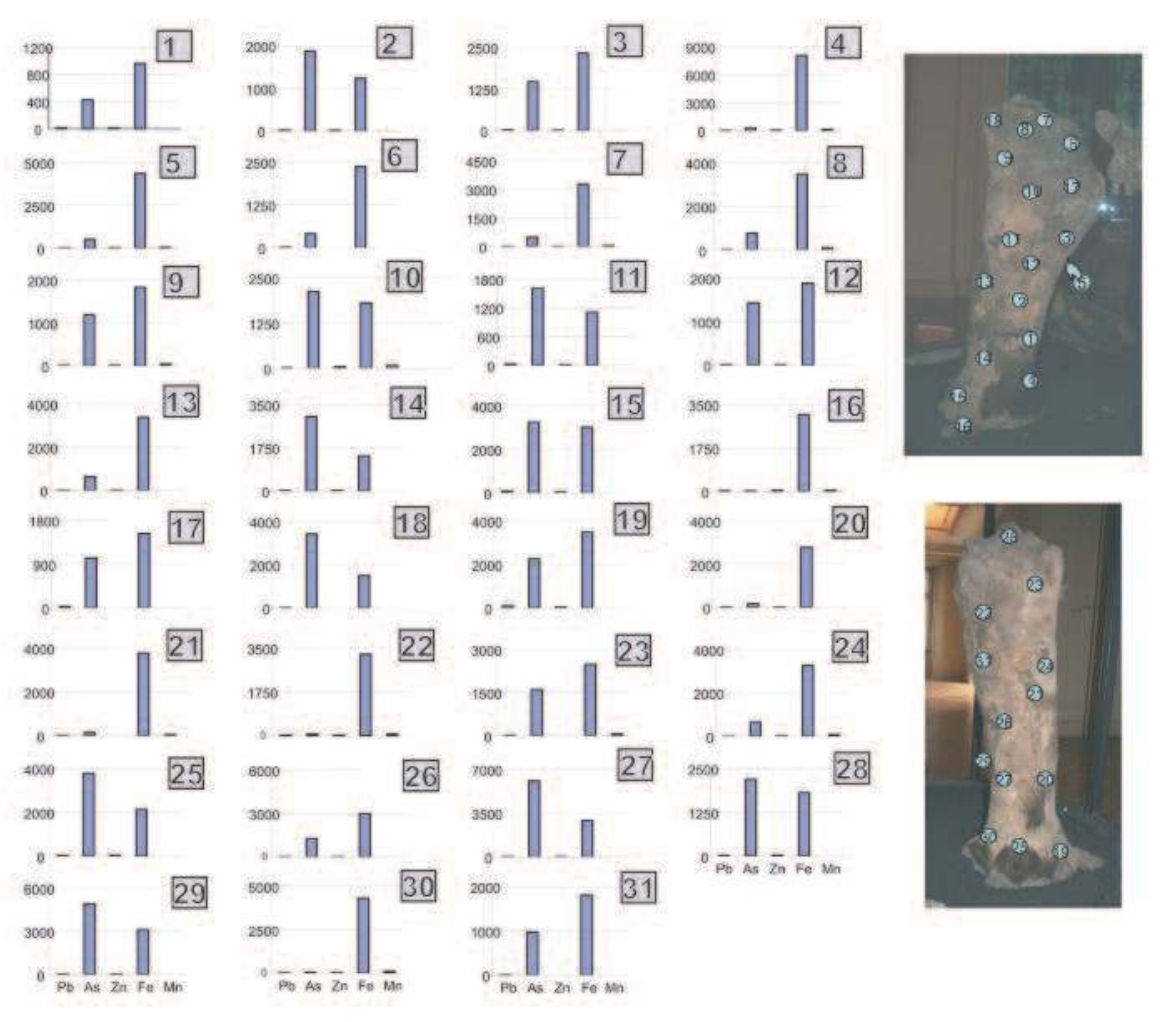

des ondes électromagnétiques de longueur d'ondes courtes et/ou longues : les ultraviolets (UV) et les infrarouges (IR) ayant pour conséquence des dégradations sur le patrimoine. On distingue, d'une part, des dégradations photothermiques dues aux ondes électromagnétiques de grande longueur d'ondes (IR) qui ont pour conséquence d'augmenter la température. D'autre part, les dégradations photochimiques sont dues aux ondes électromagnétiques de courte longueur d'ondes (radiations UV et visibles jusqu'à $550 \mathrm{~nm}$ environ). Ces ondes courtes possédant le même ordre de grandeur que les énergies d'activation des molécules composant un grand nombre de matériaux organiques, l'effet d'une irradiation (d'un éclairement) aura pour conséquence une ou des transformations dans leur agencement.

Le résultat sera des variations irréversibles de chromaticité (décoloration) et un affaiblissement de la résistance mécanique accentué par la présence d'oxygène (photo-oxydation) (Ezrati, 1998). Ces phénomènes sont donc directement liés à l'exposition lumineuse.

Les normes d'exposition à la lumière prennent aussi en compte l'intensité de l'éclairage et la durée de l'exposition lumineuse. L'intensité est adaptée à la sensibilité du matériau. Dans le cas particulier du mammouth de Lyakhov, la peau et les phanères (poils) sont des matériaux d'origine organique qui sont jugés comme extrêmement sensibles selon la classification de l'ICC (Institut canadien de Conservation) sur la base de la laine bleue. L'intensité doit être inférieure à 50 lux. La loi de réciprocité de l'action photochimique, appelée loi de Bunsen-Roscoe, indique que les dommages sont le résultat du produit de l'éclairement par la durée de l'exposition (Ezrati, 2002). La lumière a un effet cumulatif et la dose d'exposition (DTE), exprimée en million de lux/heure (Mlxh), importe. Par exemple, une exposition de 50 lux pendant 1000 heures correspond à 0,05 Mlxh. Si on considère qu'une année représente en moyenne 3000 heures d'exposition (un mois égale environ 250 heures), les recommandations donnent une exposition annuelle de 12500 lux/heure pour des objets extrêmement sensibles (Ezrati, 2002). position spectrale de la lumière, et enfin, le niveau la durée de l'éclairement. Les sources de lumière, qu'elles soient naturelles ou artificielles, produisent 


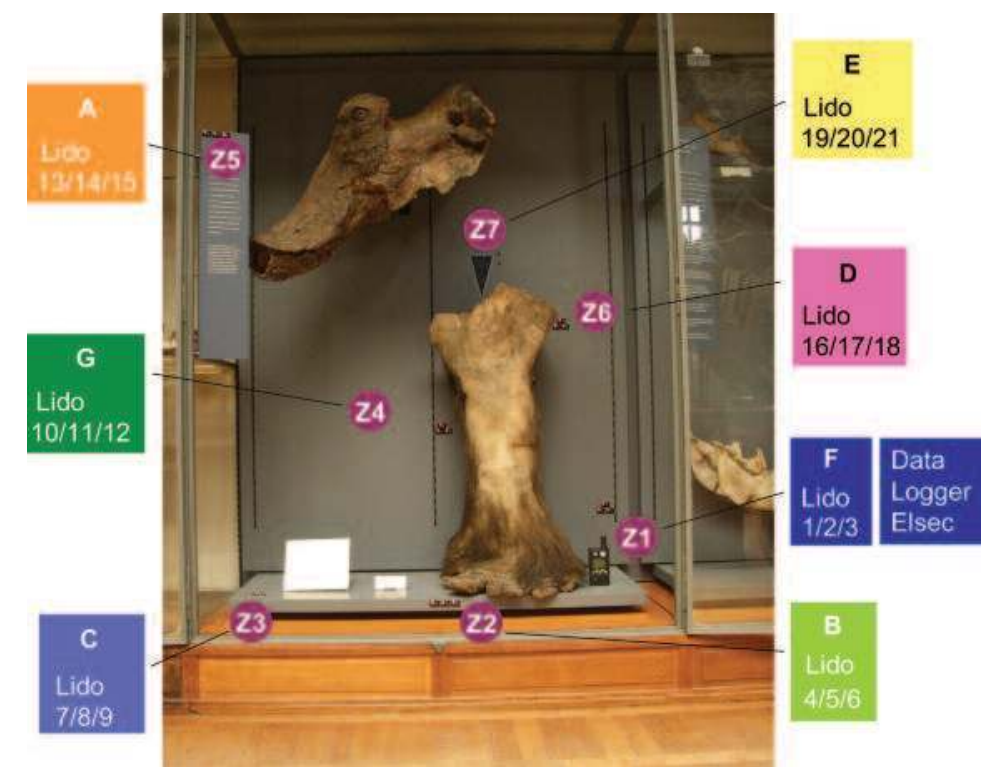

Localisation du Datalogger et des 7 zones choisies dans la vitrine 86 pour la position des $L C U \circledast$ ( 1 à 21 ) et des laines bleues ( $A$ à $G$ ).

(c) A. Péquignot

\section{Une exposition à risques}

Dans notre cas particulier, la patte de mammouth est soumise à deux systèmes d'éclairage, l'un naturel et l'autre artificiel. Les contraintes liées à l'orientation du bâtiment doivent être prises en considération pour ce constat. L'axe longitudinal du bâtiment de la galerie d'Anatomie comparée et de Paléontologie est orienté Est/Nord-Est Ouest/Sud-Ouest, en bordure de la rue Buffon, avec le pavillon de tête situé côté Seine (Est/ Nord-Est). La galerie des vertébrés fossiles, située au premier étage, est excessivement lumineuse en raison, d'une part, de sa verrière qui laisse pénétrer une lumière naturelle zénithale non filtrée en IR et en UV, car elle ne possède aucun moyen de régulation (filtre, traitement du verre, store...). Et d'autre part, la présence de 7 fenêtres de chaque côté de la nef au-dessus des vitrines murales, n'ayant pas de système d'occultation, offre une lumière et une chaleur conséquentes dans la galerie. Les vitrines périphériques consacrées à l'évolution des proboscidiens sont adossées au mur nord (côté Jardin des plantes) et sont orientées par conséquent vers le Sud. Outre l'éclairage zénithal délivré par la verrière, la vitrine 86 est soumise à l'éclairage provenant des fenêtres sud. En plus d'être soumis à la lumière naturelle, les spécimens sont exposés à une lumière artificielle produite par une coursive de néons (lampes à décharge) présents sur la partie antérieure des vitrines. Ces sources lumineuses ne possèdent également aucun système d'atténuation ou de filtration.
Les conditions de température et d'hygrométrie de cette galerie sont loin d'être idéales, comme le prouvent les valeurs enregistrées par le service de la GTB (Gestion Technique du Bâtiment, données transmises par Michel Garmier) via une sonde fixée sous la coursive de cette galerie, près du mur donnant sur le jardin, à peu près à mi-distance des extrémités de la nef. Le bâtiment n'est pas climatisé et la verrière ainsi que le chauffage/ventilation par air pulsé, rendent impossible toute tentative de régulation. Des écarts très importants de température sont constatés entre le jour et la nuit surtout l'été (la chaleur emmagasinée dans l'espace mal ventilé entre les deux épaisseurs de la verrière chauffe les espaces de la galerie). Il n'est pas rare en après-midi estival que la température atteigne $26^{\circ} \mathrm{C}$ dans la nef et dépasse $35^{\circ} \mathrm{C}$ sur la coursive du $2^{\mathrm{e}}$ étage. En revanche, la température est plus constante l'hiver $\left(17\right.$ à $19^{\circ} \mathrm{C}$ en moyenne), sauf par grand froid. Des températures extrêmes ont été enregistrées à quelques mois d'intervalle donnant l'ampleur des variations possibles : $32^{\circ} \mathrm{C}$ le 8 août 2004 à 15 h et $13^{\circ} \mathrm{C}$ le 20 décembre de la même année à $10 \mathrm{~h}$. Il s'agit là de valeurs rarissimes, mais des fluctuations nycthémérales et saisonnières de l'ordre de 10 à $12^{\circ} \mathrm{C}$ sont courantes. La patte du mammouth dans sa vitrine subit-elle les mêmes conditions environnementales, ce qui laisserait présager des dégradations irréversibles?

\section{La mise en place d'un suivi}

Les conditions climatiques de la vitrine 86 ont été étudiées sur une période de 9 mois (juin 2007 à février 2008), consistant en un suivi de la température,

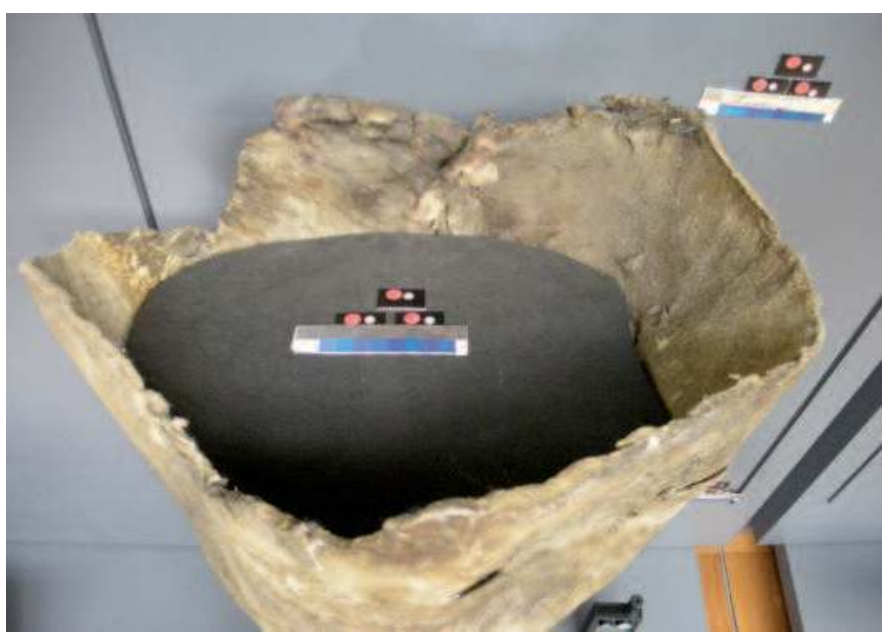

Présentation du dispositif (trois $\mathrm{LCU} \circledast$ et une laine bleue) posé sur le dessus du rembourrage de la patte (zone 7). ๑) A. Péquignot 


\section{Évolution de la couleur des pigments en fonction de la lumière}

Afin d'apprécier de la manière la plus précise la lumière reçue par la surface pigmentée (laine bleue ou $\left.\mathrm{LCU}^{\circledR}\right)$, des relevés colorimétriques sont effectués en utilisant le système CIELab* créé en 1976 par la Commission Internationale de l'Éclairage (CIE) comme un modèle absolu, indépendant du matériel. Dans ce modèle colorimétrique, une couleur est représentée par trois valeurs : L (la luminance), a et b qui sont deux gammes de couleurs respectivement a (couleurs du vert au rouge) et $\mathrm{b}$ (du bleu au jaune). Le modèle CIELab* couvre ainsi l'ensemble du spectre visible par l'oeil humain. La comparaison de deux couleurs se fait par la mesure du $\Delta \mathrm{E}^{*} \mathrm{ab}$ correspondant à la distance euclidienne entre ces deux couleurs dans l'espace colorimétrique CIELab*. Le calcul de $\Delta \mathrm{E}^{*}$ ab permet de répondre aux limites de la perception par l'œil humain : $<3$ (pas de différence perceptible), 3-6 (différence légèrement perceptible), et $>6$ (différence clairement perceptible).

La laine bleue (ISO 105-B), méthode très utilisée dans les musées, est basée sur les mêmes principes que le $\mathrm{LCU}^{\circledR}$ à la différence que les pigments qui la composent présentent une plus grande inertie à la lumière reçue. Chaque échantillon de laine bleue

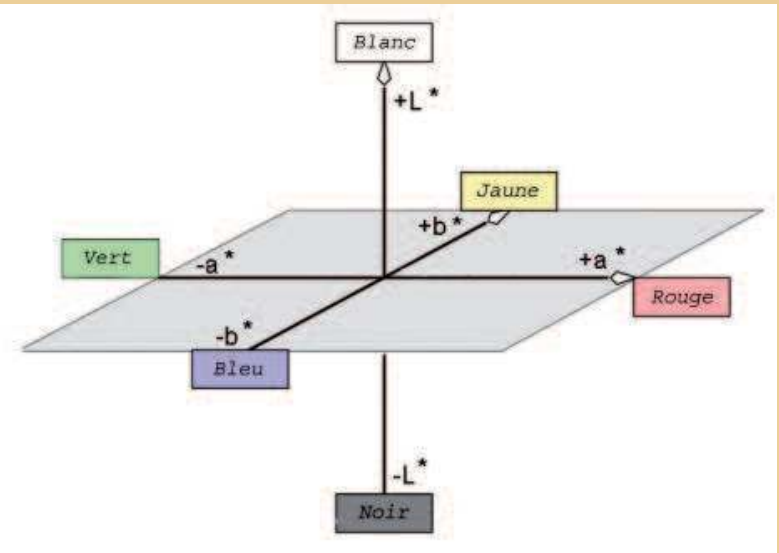

Coordonnées et attributs des couleurs dans le système CIELab* développé en 1976 par la Commission internationale de l'éclairage (CIE).

correspond à une échelle de solidité composée de huit catégories de laines teintes de référence. La première est la plus sensible à la lumière, la deuxième est approximativement deux fois moins sensible que la première, et ainsi de suite jusqu'à la huitième catégorie qui est la moins sensible. de l'humidité relative et de la lumière dans les conditions d'exposition de l'époque, de jour comme de nuit. Les paramètres thermo-hygrométriques et lumineux (éclairement et UV) ont été enregistrés électroniquement toutes les heures au moyen d'un Data Logger Elsec type 774 positionné verticalement (Z1) au plus près de la patte, afin de mettre la cellule photosensible dans le même plan que la patte du mammouth. Les relevés électroniques ont été réalisés toutes les heures. Parallèlement aux mesures thermohygrométriques, l'altération due à la lumière (éclairement et rayon ultraviolet) a été mesurée au niveau de sept zones choisies dans la vitrine : verticalement à la base de la patte (Z1), verticalement en dessous de la patte (Z2), horizontalement sur le socle (Z3), sur la cimaise à mi-hauteur (Z4) et partie haute (Z6) de la patte, sur la panneau texte (Z5) et enfin sur le dessus de la patte (Z7). Pour ce faire, deux types de détecteurs photosensibles ont été installés dans ces différentes zones : une laine bleue (ISO 105-B) et trois LightCheck Ultrasensitive ${ }^{\circledR}\left(\mathrm{LCU}^{\circledR}\right)$. La laine bleue a été à moitié couverte de papier d'aluminium afin de préserver l'état initial des différentes plages bleues au temps 0 , et d'observer leurs variations au terme de l'étude. Une fois par semaine (mardi, journée de fermeture au public), le relevé des valeurs a été effectué au moyen d'un spectrophotomètre et selon un principe basé sur le fait qu'une surface particulière est recouverte de pigments de sensibilité connue à la lumière. Ainsi, en fonction du taux d'illumination, la couleur des pigments évolue (voir encadré ci-dessus).

\section{Résultats : des conditions non adaptées}

Les résultats obtenus ( $\mathrm{n}=6033)$ montrent sur la période des moyennes de température et d'humidité relative de $19,5^{\circ} \mathrm{C}$ et de $49,4 \%$, correspondant à la zone de sécurité climatique recommandée pour les spécimens naturalisés dans les musées. Malgré ces moyennes convenables, la variation de température sur les 9 mois met en évidence deux périodes climatiques au sein de la vitrine : une période assez chaude (juin à septembre 2007) supérieure à $22^{\circ} \mathrm{C}$, et une période fraîche (septembre 2007 à janvier 2008) où les températures diminuent progressivement autour de $13^{\circ} \mathrm{C}$. Ces changements climatiques correspondent aux variations saisonnières extérieures, démontrant clairement 


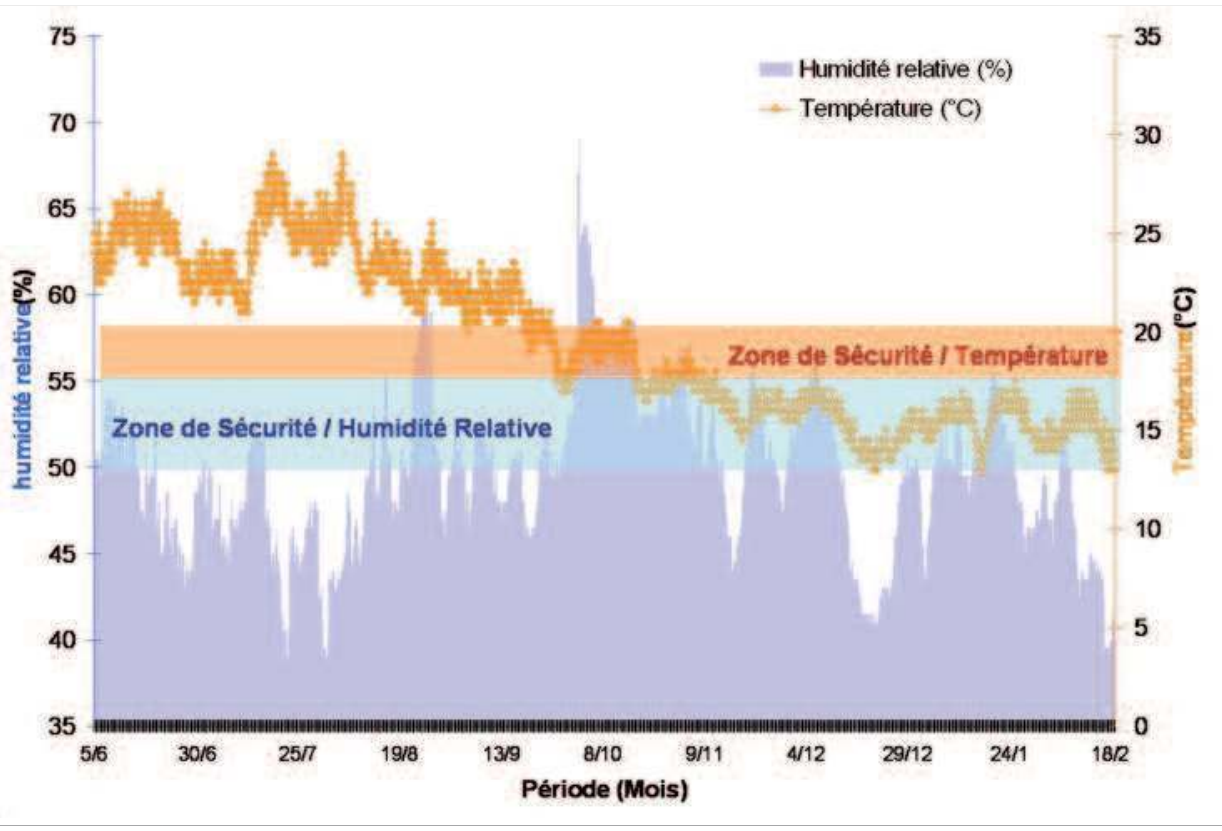

Relevés de la température et de l'humidité relative dans la vitrine 86 entre juin 2007 et février 2008.

que le bâtiment n'est pas hermétique, et que les collections exposées dans les vitrines périphériques sont directement touchées par les variations climatiques extérieures. Le climat froid et sec $\left(40 \%, 13^{\circ} \mathrm{C}\right)$ constaté peut être la cause principale de déshydratation du matériau organique, causant des dommages physiques irréversibles du spécimen comme le rétrécissement ou des torsions, sources de craquelures ou de cassures. À l'inverse, la période chaude $\left(60 \%, 22^{\circ} \mathrm{C}\right)$ est propice au développement de micro-organismes, à la condensation du matériau et à son gonflement entrainant la chute des phanères. En plus de l'identification de ces deux périodes climatiques, les variations sont, quant à elles, très importantes sur des temps relativement court ( 15 jours) : la température peut varier de 13 à $29^{\circ} \mathrm{C}$, et l'humidité relative de 36 à $69 \%$. Ces variations de grande amplitude sont néfastes pour les objets naturalisés.

Les résultats obtenus par les relevés électroniques du DataLoger montrent un éclairement élevé avec des pics en moyenne de 764 lux (juillet) ou 773 lux (août), et s'abaissant à 132 lux (décembre). Certaines maximales atteignent 1545 lux en milieu de journée du mois de juillet. Les rayonnements ultraviolets sont également élevés, en moyenne 180-200 $\mu$ Watt/Lumen. Ces valeurs montrent que les paramètres d'exposition concernant la lumière, ne répondent pas aux recommandations faites pour les objets dits « très sensibles », à savoir 50 Lux et $75 \mu \mathrm{Watt} /$ Lumen. Les données obtenues par les dosimètres $\mathrm{LCU}^{\circledR}$ répartis dans la vitrine permettent d'identifier deux secteurs d'exposition à la lumière. Le premier, regroupant les zones Z2, Z3 et Z5, présente un $\Delta \mathrm{E}^{*}$ ab qui augmente rapidement sur les 15 premiers jours de l'étude $(+30)$ traduisant une réception importante de lumière. Ce secteur correspond au devant de la vitrine, qui est le plus sujet à la lumière. Le second secteur (zones Z1, Z4, Z6, Z7) identifié présente, quant à lui, une augmentation plus faible (entre 4-11) au bout de 15 jours, mais qui se poursuit tout au long des 9 mois. Ces deux secteurs ont été également identifiés avec le système de laine bleue. La glace claire de la vitrine actuellement en place n'offre aucune protection solaire, ne limite donc pas le flux lumineux et ne filtre pas le rayonnement ultraviolet. Ces conditions d'exposition sont à l'origine de la forte décoloration et la chute importante des poils, ainsi qu’à l'état de dégradation de la peau.
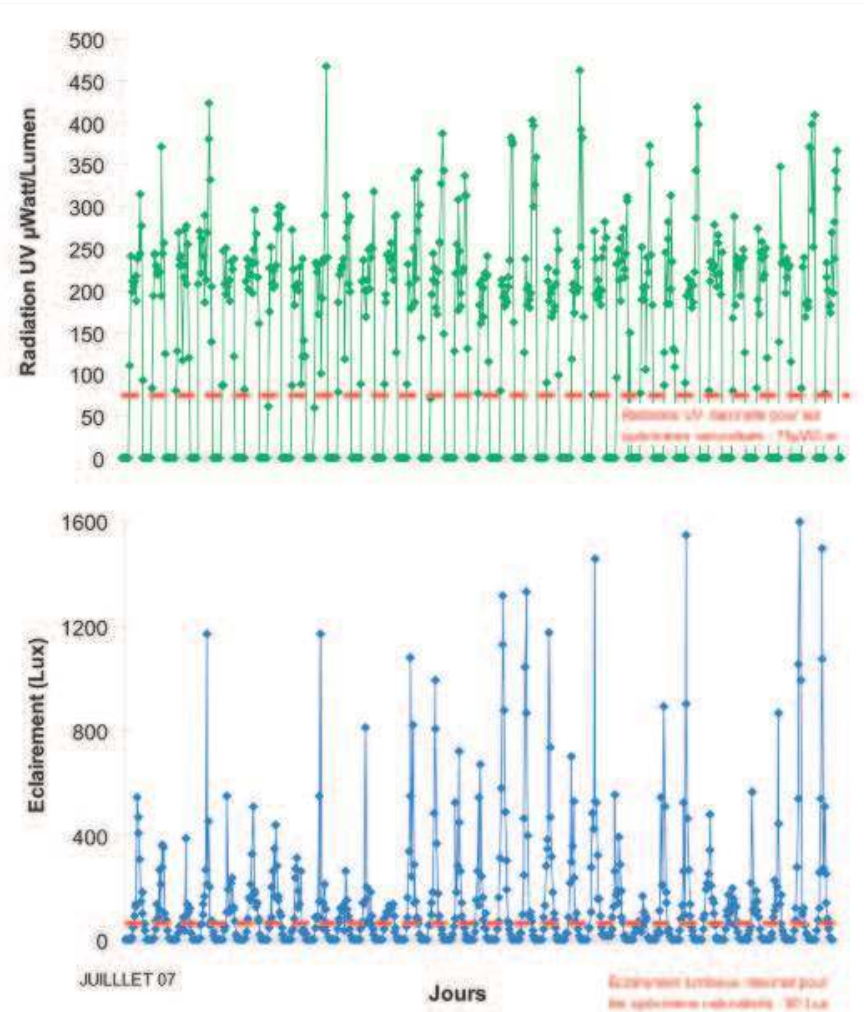

Éclairement lumineux et Radiation UV mesurés toutes les heures au cours du mois de juillet 2007. 


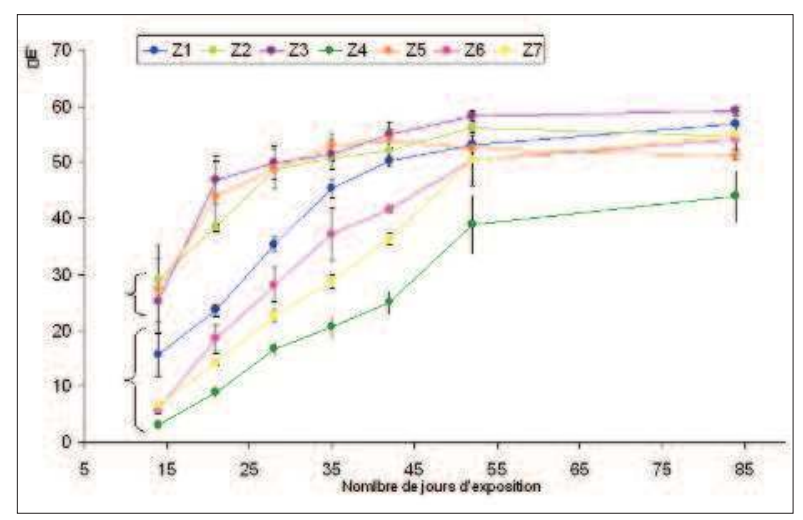

Variations de lumière reçue par $L C{ }^{\circledR}$ au niveau des différentes zones de la vitrine d'exposition (chaque donnée est la moyenne de 3 LCU par zone).

\section{Quelles solutions à court terme?}

Dans l'attente de la rénovation complète de ces galeries d'Anatomie comparée et de Paléontologie dont le schéma directeur et les travaux d'architecture et d'aménagement ont été actés il y déjà quelques années, des adaptations, à court et moyen termes, peuvent être envisagées afin d'améliorer les conditions d'exposition de ces spécimens, toujours identiques à celles de 2007, et leur conservation préventive.

Au vu des résultats de l'étude, la lumière semble être actuellement le problème majeur de l'altération, bien qu'il soit probable que les conditions de rangement en milieu chaud et sec évoquées plus haut ait été le facteur principal de la chute des phanères de ces spécimens de peau non tannés. Aujourd'hui, les moyens de protection à mettre en œuvre doivent répondre à une double contrainte : d'une part, assurer un contrôle lumineux et une protection solaire optimale, notamment en ce qui concerne les UV ; d'autre part, préserver « l'esprit du lieu » de cette galerie et la perception du visiteur en évitant de recourir uniquement à la lumière artificielle. Une première solution serait d'améliorer les performances du vitrage actuel par la pose d'un film autocollant de protection UV (élimine jusqu'à $99 \%$ du rayonnement UV, reflète la lumière visible $9 \%$ et des IR 14 \%), ou d'un film de contrôle solaire dont le but n'est que de réduire le flux lumineux. La seconde solution serait de mettre un vitrage feuilleté (PVB) réfléchissant qui, malgré un coût plus important, remplit les trois critères de limitation du visible et des UV, ainsi que le facteur solaire. L'évolution rapide de ce type de matériaux et de leur complexité nécessitera l'expertise des professionnels de l'éclairage avant toute prise de décision.

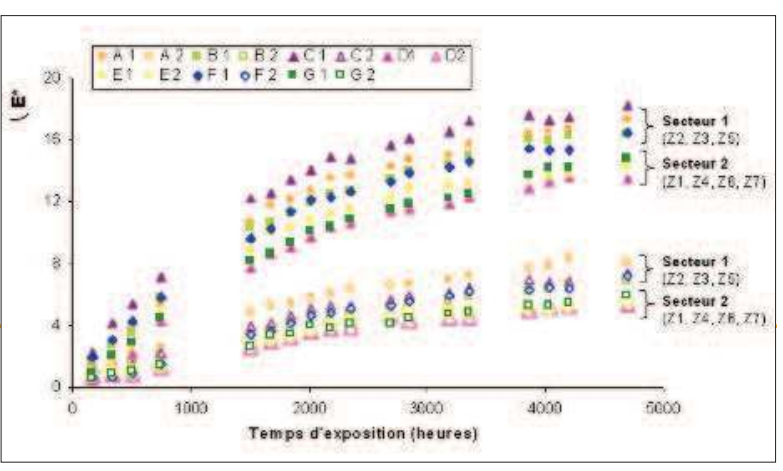

Variations du $\Delta \mathrm{E}$ pour différentes zones de la vitrine $d^{\prime}$ exposition de la patte du mammouth de Lyakhov (Laine Bleve, 2 premières plages).

Les paramètres hygrométriques sont très dépendants de l'inertie thermique du bâtiment. L'utilisation de gel de silice dans la vitrine pourrait être envisagée pour absorber l'humidité présente. Mais cette option n'est pas pertinente. Les vitrines murales étant contiguës, sans séparation, sur des longueurs de 35 mètres (la moitié de la galerie), il serait nécessaire de mettre une quantité démesurée de gel de silice pour avoir un quelconque effet sur le taux d'humidité global de ces « tunnels ». Le remplacement et le séchage du gel sous-entend une maintenance considérable qui, il faut l'avouer, ne pourra jamais être assuré. De plus, l'ouverture fréquente des vitrines par les scientifiques, du fait du double rôle des ces espaces (réserves et présentation au public), perturbe systématiquement tout équilibre hygro-thermique qui pourrait y être maintenu. En attendant le plan de rénovation du bâtiment prenant en compte lisolation des murs, la mise du spécimen en vitrine isolée et régulée pourrait être une solution.

Quant à la température, il est prévu d'installer un système de régulation $\left(20^{\circ} \mathrm{C} \pm 3\right)$ lors de la rénovation, le bâtiment ne pouvant accueillir un complexe de climatisation du fait de sa taille. D'ici cette échéance, les éclairages gagneraient à être remplacés par des dispositifs de lumière froide, ampoules et tubes à Led. Mais le problème de la diffusion de chaleur par la verrière en été reste l'inconvénient majeur et seule une ventilation frâiche entre les deux étages de verre pourrait diminuer son effet calorifère. Mais cette solution, outre un coût important, présente de nombreuse difficultés de mise en œuvre. Elle semble donc peu réaliste et relance l'opportunité d'un appel à idées.

L'exposition des peaux de mammifères sub-fossiles est peu commune. Néanmoins, certains musées présentent au public de manière permanente des peaux 
et des déjections de mylodon comme le Natural History Museum (Angleterre) et le musée de la Plata (Argentine). Dans ces deux musées, les échantillons sont exposés dans des vitrines murales entièrement fermées et isolées, ne contenant rien d'autre. La mise en exposition de la peau du mammouth de Lyakhov et sa conservation préventive se jouent à différents niveaux depuis le socle jusqu'au bâtiment en passant par la vitrine, la salle, l'étage... Par ce phénomène de poupées russes, les facteurs d'altération potentiels (acidité, composés organiques volatiles, éclairage, humidité relative, température, étanchéité, insectes et micro-organismes) s'additionnent comme autant de menaces pour les collections. Comme dans toutes les questions de conservation, ce n'est que la prise en compte globale de ces éléments qui permettra d’apporter une réponse pérenne.

Les auteurs tiennent à remercier leurs collègues $d u$ Muséum national d'Histoire naturelle, Hervé Lelièvre, Philippe Richir, Pascal Tassy (département Histoire de la Terre), Jack Thiney (direction des Collections), Michel Garmier (direction de la Rénovation) pour l'aide précieuse qu'ils ont apportée, ainsi que Cécile Héraud pour sa participation aux relevés de cette étude.

\section{Bibliographie}

Coppens, Y. Le Mammouth de l'Atrikanova (Sibérie), Bulletin du Muséum national d'Histoire naturelle, 2e série, 1958, 30 (4), pp. 402-406.

Daszkiewicz, P. Memorandum about a well-preserved mammoth from the Russian Arctic donated by Aleksander Stenbock-Fermor to Muséum national d'Histoire naturelle, Paris. Archives of natural history, 2005, 32 (1), pp. 48-52.

Debruyne R., Barreil, V. et Tassy, P. Mitochondrial cytochrome b of the Lyakhov mammoth (Proboscidea, Mammalia) : new data and phylogenetics analyses of Elephantidae. Molecular Phylogenetics and Evolution, 2003, 26, pp. 421-434.

Ezrati, J.-J. L'éclairage muséographique entre conservation et présentation : scénographie, muséologie et expographie in De Bary, M.-O. et Tobelem, J.-M. Manuel de muséographie : petit guide à l'usage des responsables de musée. Biarritz : Séguier, Option culture, 1998, pp. 121-131.

Ezrati, J.-J. Théorie, technique et technologie de l'éclairage muséographique. Paris : As Scénario, 2002, 143 p.

Krause, J., Dear, P.-H., Pollack, J.-L., Slatkin, M., Spriggs, H., Barnes, L., Lister, A.-M., Ebersberger, I. Pääbo, S. et Hofreiter, M. Multiplex amplification of the mammoth mitochondrial genome and the evolution of Elephantidae, Nature, 2006, pp. 724-727.
Mahy, M., Van Eycken, L., et Oosterlinck, A. Evaluation of uniform color spaces developed after the adoption of CIELAB and CIELUV. Color Research and Application, 1994, 19 (2) pp. 105-121.

Tassy, P. Vollossovitch et le mammouth de la grande île Liakhov. La vie au temps des mammouths, Pour la Science, 2004, avril/juin, p. 29.

Tassy, P. Das Liakhow-Mammut eine Mammutmumie aus dem sibirischen Permafrost in : Mumien. Der Traum vom ewigen Leben, Wieczorek, A., Tellenbach, M. et Rosendahl,W. Mannheim : Reiss-Engelhorn-Museen, 2007, pp. 298-299.

Van-Praët, M. Les galeries de Paléontologie et d'Anatomie comparée : vers une restitution, La Revue du musée des Arts et Métiers, 1999, n²6, pp. 55-57.

Vollosovitch, K.-A. Le Mammouth de lî̀le Bolchoï Liakovsky (îles de la Nouvelle Sibérie). Verhandlungen der russisch-kaiserlichen mineralogischen Gessellschaft zu St Petersburg, 1914, 2e série, pp. 305-338. 